Descargo de responsabilidad: las opiniones expresadas en los manuscritos son responsabilidad exclusiva de los autores. No necesariamente reflejan las opiniones de la editorial ni la de sus miembros.

1 CENIRO

\title{
Mentalidad de crecimiento, clave para un óptimo funcionamiento de Centros de Recursos para la Educación Inclusiva
}

Growth Mindset, key to an optimal functioning of Resourse Centers for Inclusive Education

Recibido: 05/10/2021

Publicado: 20/01/2022

Filiación institucional de los autores

Maestría en Neurociencia con énfasis en Neurocognición

Universidad de San Carlos de Guatemala

Ana Lissette Bran Solórzano

braso.lissette@gmail.com

https://orcid.org/0000-0002-8265-2007

Iris Maribel Canel Pinto

Keitlyn.ailyn@gmail.com

https://orcid.org/0000-0001-5093-4184

\section{Referencia}

Mayra Nineth Lemus

manineth3@gmail.com

https://orcid.org/0000-0002-3545-1114

Concepción Yenifer Marleny Osegueda

yenifer.osegueda@gmail.com

https://orcid.org/0000-0002-1511-4030

Bran Solórzano, A. L., Canel Pinto, I. M., Lemus, M. N., \& Marleny Osegueda, C. Y. (2022). Mentalidad de crecimiento, clave para un óptimo funcionamiento de Centros de Recursos para la Educación Inclusiva. Revista Académica Sociedad Del Conocimiento Cunzac, 2(1), 25-31. DOI: https://doi.org/10.46780/sociedadcunzac.v2i1.9

\section{Resumen}

OBJETIVO: establecer la caracterización del funcionamiento de los Centros de Recursos para la Educación Inclusiva, tomando en cuenta el tipo de mentalidad que presentan los docentes. MÉTODO: el enfoque de la investigación fue descriptivo de corte trasversal con una población de 91 docentes CREI, ubicados en 14 departamentos de Guatemala. RESULTADOS: se demostró que el rango etario frecuente fue de 36 a 59 años equivale al $68.13 \%(\mathrm{~N}=62)$; el género predominante fue femenino con $73.63 \%(\mathrm{~N}=67)$, el grupo étnico ladino/mestizo lo representa el $78.02 \% \quad(\mathrm{~N}=71)$; los profesionales indicaron que la prevalencia académica es licenciatura con $54.94 \%(\mathrm{~N}=50)$; presentaba una mentalidad fija el $42.86 \%(\mathrm{~N}=39)$; y después de haber recibido estrategias neurodidácticas los resultados evidencian que $57.14 \%(\mathrm{~N}=52)$ presentó mentalidad de crecimiento. CONCLUSIÓN: La mentalidad de crecimiento es la inteligencia que prevaleció en los docentes, después de recibir estrategias neurodidácticas, les permitió planificar, desarrollar y ejecutar nuevas metodologías de aprendizaje para tener efecto positivo en los estudiantes, en el desempeño académico con actitudes auténticas y mayor confianza en sí mismos; así como nuevas formas de pensar y actuar, esto permite que el sistema educativo sea inclusivo y exista mayor permanencia de estudiantes con necesidades educativas especiales asociadas o no a discapacidad.

\section{Palabras clave}

mentalidad de crecimiento, mentalidad fija, centros de recursos para la educación inclusiva 


\section{Abstract}

OBJECTIVE: to establish the characterization of the functioning of the Resource Centers for Inclusive Education, taking into account the type of mentality presented by teachers. METHOD: the research approach was descriptive of a cross-sectional nature with a population of 91 CREI professors, located in 14 departments of Guatemala. RESULTS: it was shown that the frequent age range was from 36 to 59 years equivalent to $68.13 \%(N=62)$; the predominant gender was female with $73.63 \%(\mathrm{~N}=67)$, the Ladino/mestizo ethnic group represents $78.02 \%$ $(\mathrm{N}=71)$; the professionals indicated that the academic prevalence is bachelor's degree with $54.94 \%(\mathrm{~N}=50)$; had a fixed mentality of $42.86 \%(\mathrm{~N}=39)$; and after having received neurodydical strategies, the results show that $57.14 \%(\mathrm{~N}=52)$ presented a growth mentality. CONCLUSION: the growth mentality is the intelligence that prevailed in teachers, after receiving neurodydical strategies, allowed them to plan, develop and execute new learning methodologies to have a positive effect on students, on academic performance with authentic attitudes and greater self-confidence; as well as new ways of thinking and acting, this allows the educational system to be inclusive and there is greater permanence of students with special educational needs associated or not with disability.

\section{Keywords}

growth mindset, fixed mindset, resource centers for inclusive education

\section{Introducción}

En Guatemala, el discurso de educación para todos sigue siendo una utopía, la inclusión de las personas con discapacidad en el sistema educativo nacional ha sido abordada desde diferentes aristas, por varios años sin embargo no todos los estudiantes tienen las mismas oportunidades de aprender y la permanencia escolar es siempre un reto para la mayor parte de la comunidad educativa. Hernández \& Zil (2021), refieren en el Documento base para la implementación de los Centros de Recursos para la Educación Inclusiva al Acuerdo Gubernativo No. 225-2008 que establece a la Dirección General de Educación Especial, como la responsable de la educación especial y la encargada de velar por la aplicación correcta de las leyes y políticas para las personas con capacidades especiales, (p. 6).

El Ministerio de Educación en el año 2017 implementó los Centros de Recursos para la Educación Inclusiva -CREI-. como un programa al servicio de la comunidad educativa contribuyendo así a la ampliación de cobertura, esta estrategia pretende disminuir la brecha de acceso, permanencia de la población con necesidades educativas especiales asociadas o no a discapacidad y hacer valer los derechos de todos los estudiantes. (Hernández \& Zil, 2021, p. 6). Los CREI tienen el fin de promover la educación inclusiva fundamentada en el aprendizaje significativo, convirtiendo al docente responsable en un profesional que produce, construye conocimientos y los socializa en la comunidad educativa. El presente artículo surge por el interés de desarrollar una mentalidad de crecimiento para el óptimo funcionamiento de los Centros de Recursos para la Educación Inclusiva -CREI- En neurociencia la mentalidad de crecimiento se conoce como una estrategia de motivar y estimular el cerebro de las personas, para mejorar el rendimiento en cada una de las áreas en que éste se desarrolla y obtener placer por realizar actividades que se propone con éxito. Es sin duda una de las habilidades que manifiestan los docentes encargados, desarrollando acciones educativas en beneficio de otros docentes, pa- 
dres de familia y estudiantes con necesidades educativas especiales, así como sus destrezas y habilidades en diversas temáticas que van en aumento a partir de la formación recibida.

\section{Materiales y métodos}

Investigación de tipo descriptiva de corte transversal con una población de 91 docentes como universo total, por lo cual la información se obtuvo por medio de un instrumento de recolección de datos para el análisis de resultados. Los que se evidencian en 3 categorías, características demográficas, población beneficiada, evaluación inicial de conocimientos y evaluación con estrategias neurodidácticas.

\section{Resultados}

Tabla 1. La caracterización demográfica.

\section{Cuadro de variables Docentes Responsables CREI}

\begin{tabular}{|c|c|c|c|}
\hline \multicolumn{2}{|c|}{ Características } & \multirow{2}{*}{$\begin{array}{c}\text { Cantidad } \\
62 \\
\end{array}$} & \multirow{2}{*}{$\begin{array}{c}\% \\
68.13 \\
\end{array}$} \\
\hline Rango etario & 36-59 años & & \\
\hline & 18-35 años & 27 & 29.67 \\
\hline & 60 años o más & 2 & 2.20 \\
\hline \multirow[t]{2}{*}{ Género } & Femenino & 67 & 73.63 \\
\hline & Masculino & 24 & 26.37 \\
\hline \multirow[t]{2}{*}{ Grupo étnico } & Ladino/Mestizo & 71 & 78.02 \\
\hline & Maya & 20 & 21.98 \\
\hline \multirow[t]{6}{*}{ Idioma } & Castellano & 73 & 80.22 \\
\hline & Kaqchikel & 8 & 8.79 \\
\hline & Man & 5 & 5.49 \\
\hline & $\mathrm{K}^{\prime}$ iche' & 2 & 2.20 \\
\hline & Tzútujil & 2 & 2.20 \\
\hline & Garifuna & 1 & 1.10 \\
\hline \multirow[t]{14}{*}{ Residencia } & San Marcos & 25 & 27.47 \\
\hline & Petén & 12 & 13.19 \\
\hline & Quetzaltenango & 12 & 13.19 \\
\hline & Jutiapa & 6 & 6.59 \\
\hline & Sololá & 6 & 6.59 \\
\hline & Jalapa & 5 & 5.49 \\
\hline & Huehuetenango & 4 & 4.39 \\
\hline & Quiché & 4 & 4.40 \\
\hline & Suchitepéquez & 4 & 4.40 \\
\hline & Totonicapán & 4 & 4.40 \\
\hline & Alta Verapaz & 3 & 3.30 \\
\hline & Guatemala & 3 & 3.30 \\
\hline & Zacapa & 2 & 2.20 \\
\hline & Izabal & 1 & 1.09 \\
\hline \multirow[t]{4}{*}{ Escolaridad } & Licenciatura & 50 & 54.94 \\
\hline & Nivel Medio & 19 & 20.88 \\
\hline & Técnico Universitario & 11 & 12.09 \\
\hline & Maestría & 11 & 12.09 \\
\hline
\end{tabular}

Fuente: elaboración propia. Instrumento de recolección de datos. 
En la tabla anterior los docentes encargados de los CREI, el $68.13 \%(\mathrm{~N}=62)$ pertenece al rango etario de 36 a 59 años; el 73.63\% ( $\mathrm{N}=67)$ es de género femenino; el grupo étnico ladino/mestizo que representa el $78.02 \%(\mathrm{~N}=71)$; y su escolaridad el $54 \%(\mathrm{~N}=50)$ en el grado académico de licenciatura, el idioma predominante es el castellano con el $80.22 \%(\mathrm{~N}=73)$, y residentes en los 14 departamentos del país, con cobertura del programa.

Tabla 2. La caracterización de la población beneficiada. Cuadro de variables Estudiantes Beneficiados

\begin{tabular}{|c|c|c|c|}
\hline \multicolumn{2}{|c|}{ Características } & \multirow{2}{*}{$\begin{array}{c}\text { Cantidad } \\
2,480\end{array}$} & \multirow{2}{*}{$\begin{array}{c}\% \\
63.73\end{array}$} \\
\hline \multirow{3}{*}{ Rango etario } & 6 a 9 años & & \\
\hline & 10 a 12 años & 1,346 & 34.58 \\
\hline & 13 a 16 años & 66 & 1.69 \\
\hline \multirow{2}{*}{ Género } & Masculino & 2,337 & 60.05 \\
\hline & Femenino & 1,555 & 39.95 \\
\hline \multirow{3}{*}{ Nivel Educativos } & Primaria & 2,838 & 72.82 \\
\hline & Preprimaria & 943 & 24.23 \\
\hline & Medio & 111 & 2.85 \\
\hline \multirow{9}{*}{$\begin{array}{l}\text { Necesidades } \\
\text { Educativas } \\
\text { Especiales } \\
\text { asociadas o no a } \\
\text { Discapacidad }\end{array}$} & Totalidad a nivel país & 3,892 & 100 \\
\hline & $\begin{array}{l}\text { Necesidades educativas } \\
\text { especiales (no D) }\end{array}$ & 1,556 & 39.98 \\
\hline & Discapacidad Intelectual & 1,101 & 28.29 \\
\hline & Discapacidad auditiva & 361 & 9.28 \\
\hline & Discapacidad motora & 301 & 7.73 \\
\hline & Discapacidad visual & 253 & 6.50 \\
\hline & Múltiple & 141 & 3.62 \\
\hline & $\begin{array}{l}\text { Trastornos generalizados del } \\
\text { desarrollo }\end{array}$ & 141 & 3.62 \\
\hline & Talla Pequeña Acondroplasia & 38 & 0.98 \\
\hline
\end{tabular}

Fuente: estadística MINEDUC/Digeesp 2021.

En la tabla numero 2 corresponde a la caracterización de la población beneficiaria, el grupo etario de 6-9 años pertenece al 63.73\% $(\mathrm{N}=2,480)$ el nivel primario del Sistema Educativo Nacional prevaleció con el $72.82 \%(\mathrm{~N}=2,838)$; género masculino representa el $60.05 \%$ $(\mathrm{N}=2,337)$, los niños con necesidades educativas especiales asociadas o no a discapacidad al $39.98 \%(\mathrm{~N}=1,556)$ y la discapacidad que prevalece con el $28.29 \%(\mathrm{~N}=1101)$ es la intelectual. 
Gráfica 1. Evaluación inicial y con estrategias Neurodidácticas.

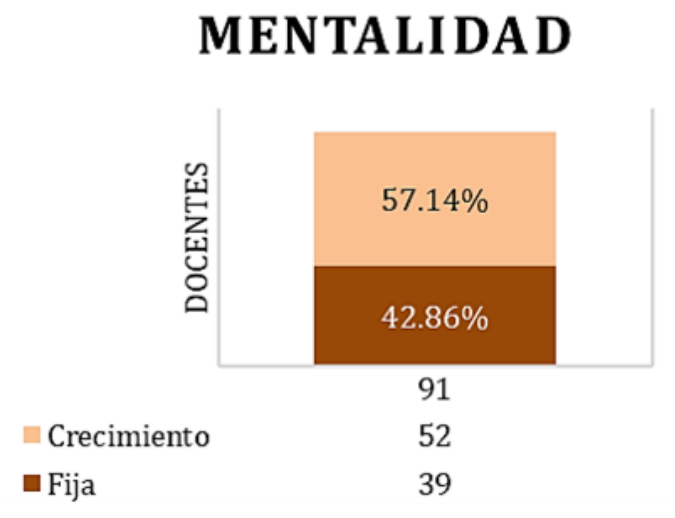

Fuente: elaboración propia. Instrumento de recolección de datos.

En la gráfica anterior se puede observar que antes de pertenecer al programa CREI el $42.86 \%$ $(\mathrm{N}=39)$ de los docentes se encontraban con una mentalidad fija y después de la formación neurodidáctica, el $57.14 \%(\mathrm{~N}=52)$ de la población mostró una evolución significativa en la mentalidad de crecimiento.

\section{Discusión}

La Educación en Guatemala a través de la historia ha evidenciado problemáticas en los estudiantes los cuales se puede mencionar: fracaso, deserción, bajo rendimiento y exclusión escolar; baja autoestima y una diversidad de necesidades educativas especiales.

El Ministerio de Educación -MINEDUC-, en el plan estratégico 2016-2020 estableció municipios priorizados en 12 departamentos, donde implementó la apertura de 37 "Centros de Recursos para la Educación Inclusiva" - CREI- contribuyendo así a la ampliación de la cobertura y permanencia de los estudiantes dentro del Sistema Educativo Nacional, como lo indica (Hernández \& Zil, 2021, p. 6).

De acuerdo a la investigación realizada para establecer la caracterización del funcionamiento y tener en cuenta la mentalidad de crecimiento que presentan los docentes responsables de los centros de recursos; se encontró las características sociodemográficas más predominantes en los docentes encargados de los CREI, el 68.13\% pertenece al rango etario de 36 a 59 años; la mayor parte fueron del género femenino con el $73.63 \%$; el grupo étnico ladino/mestizo que representa el $78.02 \%$; y la escolaridad el $54 \%$ en el grado académico de licenciatura, el idioma predominante es el castellano con el $80.22 \%$.

La caracterización de la población beneficiaria por el programa CREI, arroja: al grupo etario de 6-9 años con el 63.73\%; el nivel primario del Sistema Educativo Nacional con el 72.82\%; 
el $60.05 \%$ representa al género masculino, el $39.98 \%$ a los niños con necesidades educativas especiales asociadas o no a discapacidad y la discapacidad que prevalece con el $28.29 \%$ es la Intelectual.

Dweck, (2006) en su teoría de la mentalidad de crecimiento, en las personas atribuyen el éxito al esfuerzo, es decir conciben que las habilidades pueden cultivarse mediante la práctica constante. En la gráfica número uno se observa que antes de pertenecer al programa CREI el $39 \%$ de los docentes se encontraban con una mentalidad fija y después de la formación neurodidáctica, el $52 \%$ de la población mostró una evolución significativa en la mentalidad de crecimiento.

Los docentes encargados de los CREI, después de recibir la formación en estrategias neurodidácticas, adquieren una mentalidad de crecimiento y se refleja en la atención de la población beneficiada; se puede afirmar que, al efectuar técnicas de mentalidad de crecimiento, se promueven los cambios intelectuales, de habilidades y destrezas que influyen en lo académico y en lo emocional.

\section{Referencias}

Dweck, C. (2006). Mindset. "La actitud del éxito". https://corladancash.com/wp-content/ uploads/2020/01/Mindset-La-actitud-del-exito-CAROL-S.-DWECK.pdf

Hernández, I., \& Zil Roca, E. (2021). Guía para el funcionamiento del Centro de Recursos para la Educación Inclusiva. Características de la Educación Inclusiva. Guatemala, Guatemala. (p. 6) https://drive.google.com/file/d/1fEkrgecxvMkt3G43Vj6De8fHuH4dB8uZ/view?us$\mathrm{p}=$ sharing

Hernández , I., \& Zil Roca , E. (2021). Documento base para la implementación de los Centros de Recursos para la Educación Inclusiva Características de la Educación Inclusiva. Guatemala, Guatemala. (p. 6) https://drive.google.com/file/d/1fDUpSRmuuc7zeAsZW4Dik87RctPPFeXm/view?usp=sharing

\section{Sobre los autores}

Grupo de investigadores de la maestría en Neurociencias con énfasis en Neurocognición del Centro Universitario de Zacapa.

Financiamiento de la investigación

Con recursos propios de los investigadores

\section{Declaración de intereses}

Declaran no tener ningún conflicto de intereses, que puedan haber influido en los resultados obtenidos o las interpretaciones propuestas. 


\section{Declaración de consentimiento informado}

El estudio se realizó respetando el Código de ética y buenas prácticas editoriales de publicación.

\section{Derechos de uso}

Copyright@ 2022 por Ana Lissette Bran Solórzano, Iris Maribel Canel Pinto, Mayra Nineth Lemus y Concepción Yenifer Marleny Osegueda.

Este texto está protegido por una licencia Creative Commons 4.0

Este texto está protegido por la Licencia Creative Commons Atribución 4.0 Internacional.

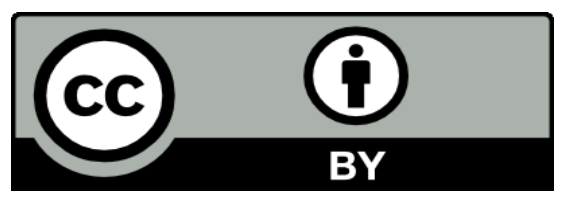

Este texto está protegido por una licencia

Creative Commons 4.0.

Es libre para compartir, copiar y redistribuir el material en cualquier medio o formato y adaptar el documento, remezclar, transformar y crear a partir del material para cualquier propósito, incluso comercialmente, siempre que cumpla la condición de atribución: debe reconocer el crédito de una obra de manera adecuada, proporcionar un enlace a la licencia, e indicar si se han realizado cambios. Puede hacerlo en cualquier forma razonable, pero no de forma tal que sugiera que tiene el apoyo del licenciante o lo recibe por el uso que hace. 